\title{
The Effects of Deformed Projectile in Threshold Anomaly and Fusion Reaction for ${ }^{19} \mathbf{F}+{ }^{208} \mathrm{~Pb}$ System
}

\author{
C. J. Lin, ${ }^{*}$ H. Q. Zhang, Z. H. Liu, J. C. Xu, and F. Yang \\ China Institute of Atomic Energy, P.O. Box 275(10), Beijing 102413, China
}

Received: November 13, 2001; In Final Form: November 13, 2001

Angular distributions of elastic scatterings for ${ }^{19} \mathrm{~F}+{ }^{208} \mathrm{~Pb}$ system have been measured at six energies around the Coulomb barrier. It is found that the real and imaginary potentials show pronounced energy dependence in terms of the phenomenological optical model analysis. The real parts of potentials extracted from the fusion data are similar to those from the elastic data. A comparison with the neighbouring systems of ${ }^{16} \mathrm{O}+{ }^{208} \mathrm{~Pb}$ and ${ }^{16} \mathrm{O}+{ }^{209} \mathrm{Bi}$ reveals that the ${ }^{19} \mathrm{~F}$ deformations play an important role in the fusion reaction. Based on the deformed and energy dependent barrier penetration model, the calculated fusion cross sections and mean-square spin distribution agree with the experimental data well. Moreover, it is indicated that the enhancement of fusion cross sections attributes to the dynamic polarization effects around the barrier, and to the static deformation effects further below the barrier.

\section{Introduction}

It is well known that the threshold anomaly (TA) comes from the coupled-channels (CC) effects and plays an important role in heavy ion reaction at the energies around the Coulomb barrier. ${ }^{1,2}$ The existence of the TA in the interacting potential for the elastic scattering has been formed for many systems. This anomaly shows up as a localized peak in the real part of the interacting potential around the Coulomb barrier and it is associated with the decrease of the imaginary part of the interacting potential in the same energy range. It has been shown that this correlation between the real and imaginary parts of the interacting potential is due to causality and consequently that they obey the dispersion relation.

Due to the difficulty to calculate the dynamic polarization potential in theory, TA is still a purely experimental matter up to now. Most of systems studied are spherical or near spherical. Very little work has been done for well deformed system ${ }^{3}$ with attempt to understand the effects of nuclear structure, especially for the deformed projectile. In this work, the ${ }^{19} \mathrm{~F}+{ }^{208} \mathrm{~Pb}$ system was chosen because ${ }^{19} \mathrm{~F}$ nucleus has quite large static deformations $\left(\beta_{2}=0.44\right.$ and $\left.\beta_{4}=0.14\right){ }^{4}$ The CC effects in this system should be pronounced and appreciably influence on the fusion channel. Since the neighboring systems of ${ }^{16} \mathrm{O}+{ }^{208} \mathrm{~Pb}$ and ${ }^{16} \mathrm{O}+{ }^{209} \mathrm{Bi}$ are well investigated, ${ }^{5-7}$ it is interesting to make a comparison with these systems. Furthermore, the fusion data of ${ }^{19} \mathrm{~F}+{ }^{208} \mathrm{~Pb}$ system are available in the literatures. ${ }^{8,9}$ It is meaningful to compare these data with the calculations of barrier penetration model (BPM) modified by the deformations and energy dependence.

\section{Experimental Procedure}

The experiment was carried out at $\mathrm{HI}-13$ tandem accelerator of China Institute of Atomic Energy, Beijing. $\mathrm{A}^{208} \mathrm{~Pb}$ target of thickness about $100 \mu \mathrm{g} / \mathrm{cm}^{2}$ evaporated onto a $20 \mu \mathrm{g} / \mathrm{cm}^{2}$ carbon foil backing was bombarded by a collimated ${ }^{19} \mathrm{~F}$ beam. The beam energies were $88,91,93,96,98$, and $102 \mathrm{MeV}$, respectively. The reaction products were detected by three pairs of $\Delta E-E$ telescope detectors with the total energy resolution less than $1.3 \%$. A typical spectrum cut by the $Z=9$ banana in the $\Delta E-E$ matrix at $E_{L}=102 \mathrm{MeV}$ and $\theta_{L}=86^{\circ}$ is shown in Figure 1 . A Si(Au) surface barrier detector was mounted at $-42.4^{\circ}$ with respect to the beam direction as a monitor to detect the elastic scatterings. All the reaction cross sections were normalized to the monitor counts, assuming that the elastic cross sections are

*Corresponding author. E-mail: cjlin@iris.ciae.ac.cn. FAX: +8610-6935-7787. equal to the Rutherford cross sections at the forward angles.

The energy resolutions of the detectors allow to separate all the $E_{x} \geq 1.3457 \mathrm{MeV}$ states of ${ }^{19} \mathrm{~F}$ and all the excitation states of ${ }^{208} \mathrm{~Pb}$ from ground states, while the two lower excitation states $0.1099 \mathrm{MeV}$ and $0.1971 \mathrm{MeV}$ of ${ }^{19} \mathrm{~F}$ cannot be resolved from the measured elastic scatterings. Thus the elastic spectra include the inelastic scatterings of these two low-lying excitation states. The differential elastic scattering cross sections normalized to the Rutherford cross sections for all energies are shown in Figure 2. The overall error is $3 \%$ for the forward angles and gradually increases to $10 \%$ for the backward angles.

\section{Energy Dependent Potentials}

3.1. Optical Model Analysis of Elastic Scatterings. In this work the phenomenological analyses are utilized to get the polarization optical potentials. Since the low-lying excited states are not resolved, the coupled-channels code ECIS $95^{10}$ is employed to fit the elastic scattering angular distributions in order to get the pure elastic scattering potentials. In these calculations only the couplings between the $E_{x}=0.1971 \mathrm{MeV}, J^{\pi}=5 / 2^{+}$ state and ground state which belong to the $K=1 / 2^{+}$ground state rotational band are taken into account. The same optical potentials are assumed for these two coupling nuclear states. Another excitation state $E_{x}=0.1099 \mathrm{MeV}, J^{\pi}=1 / 2^{-}$which belongs to $K=1 / 2^{-}$band is not included, because the strength coupled to the ground state band is very weak.

In the calculations, the Woods-Saxon shapes of the real and imaginary potentials are kept the same $\left(r_{0 v}=r_{0 w}=r_{0}\right.$,

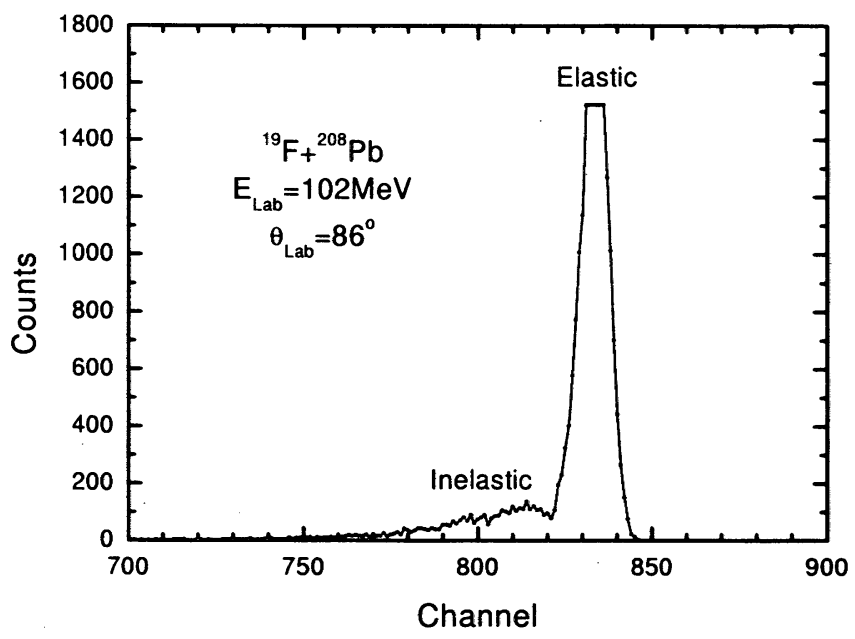

Figure 1. A typical elastic and inelastic spectrum. 
TABLE 1: The sensitive radii and the results of the best fits obtained at $r_{0}=1.24 \mathrm{fm}$ and $a_{0}=0.53 \mathrm{fm}$ for elastic scatterings.

\begin{tabular}{ccccccc}
\hline $\begin{array}{c}E_{\text {lab }} \\
/ \mathrm{MeV}\end{array}$ & $\begin{array}{c}R_{S V} \\
/ \mathrm{fm}\end{array}$ & $\begin{array}{c}R_{S W} \\
/ \mathrm{fm}\end{array}$ & $\begin{array}{c}-V_{0} \\
/ \mathrm{MeV}\end{array}$ & $\begin{array}{c}-W_{0} \\
/ \mathrm{MeV}\end{array}$ & $\chi^{2} / p t$ & $\begin{array}{c}\sigma_{R} \\
/ \mathrm{mb}\end{array}$ \\
\hline 102 & 12.86 & 13.16 & 73.2 & 44.5 & 7.67 & 1169 \\
98 & 12.62 & 12.75 & 77.2 & 23.4 & 10.5 & 897.2 \\
96 & 12.58 & 12.89 & 77.5 & 22.5 & 27.1 & 838.3 \\
93 & 12.50 & 12.20 & 85.1 & 10.2 & 14.2 & 673.2 \\
91 & 12.32 & 12.12 & 88.6 & 3.44 & 8.87 & 579.9 \\
88 & 12.24 & & 78.4 & 1.57 & 1.25 & 512.0
\end{tabular}

$\left.a_{\nu}=a_{w}=a_{0}\right)$. In order to assess the influences of the potential parameters, two sets of fits are performed with the fixed radius parameters $r_{0}=1.20 \mathrm{fm}$ and $r_{0}=1.24 \mathrm{fm}$, respectively. For each set, a grid search is made on the different diffuseness parameters $\left(a_{0}=0.43,0.48,0.53,0.58,0.63,0.68 \mathrm{fm}\right)$ to obtain the best $V_{0}$ and $W_{0}$ values with the minimum $\chi^{2}$. It is found that the $\chi^{2}$ values reach their minimum when $r_{0}=1.24 \mathrm{fm}$ and $a_{0}=0.53 \mathrm{fm}$ for all energies. The results of the best fits in this case are illustrated in Figure 2 as the solid lines and the parameters are listed in Table 1. All the lines of the real or imaginary potentials with different parameters converge at a certain distance, namely sensitive radius $\left(R_{S}\right)$. At the $R_{S}$, the potentials have a minimum uncertainty and are nearly independent of geometrical shapes. It is realized that the $R_{S}$ values depend on the energies and have some differences between the real and imaginary parts of potentials. For consistency, $R_{S}$ are taken as $12.5 \mathrm{fm}$ for both real and imaginary parts of potentials at all energies. The real and imaginary parts of potentials at the $R_{S}\left(V_{S}\right.$ and $\left.W_{S}\right)$ are illustrated in Figure 3 with the circle symbols. The errors are derived from the $\chi^{2}$ values. It is clear that the potentials are energy dependent around the Coulomb barrier, as the typical TA behaviour.

3.2. Extract TA Parameters in Linear Model. For convenience, the linear schematic model of the two straight line segments is utilized to describe the TA behaviour. ${ }^{1,2} W_{S}(E)$ is

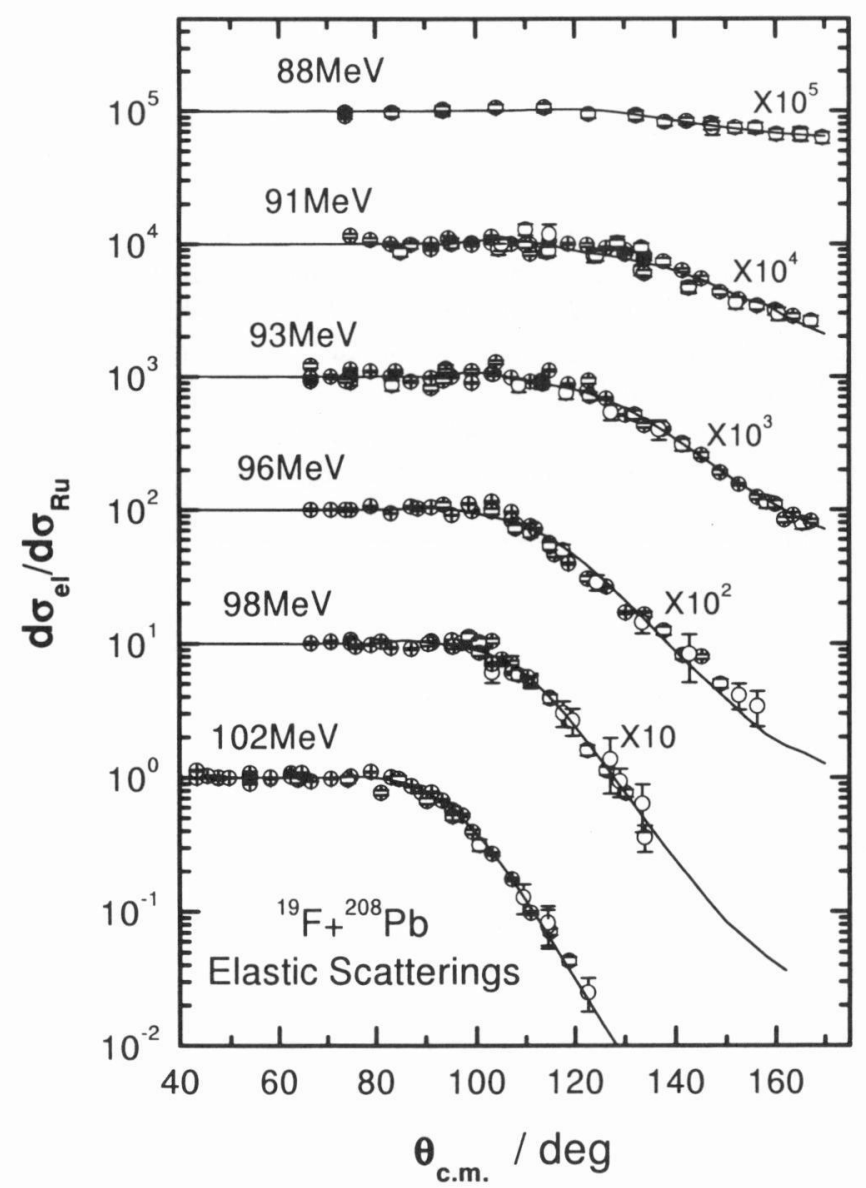

Figure 2. Angular distributions of elastic scatterings. defined as

$$
W_{S}(E)=\left\{\begin{array}{lc}
0 & E \leq E_{a} \\
W_{0 S} \frac{E-E_{a}}{E_{b}-E_{a}} & E_{a}<E<E_{b}, \\
W_{0 S} & E \geq E_{b}
\end{array},\right.
$$

and according to the dispersion relation, $V_{S}(E)$ is

$$
\begin{aligned}
V_{S}(E) & =V_{0 S}+\Delta V_{S}(E) \\
& =V_{0 S}-\left(W_{0 S} / \pi\right)\left[\varepsilon_{a} \ln \left|\varepsilon_{a}\right|-\varepsilon_{b} \ln \left|\varepsilon_{b}\right|\right] .
\end{aligned}
$$

Here $\varepsilon_{i}=\left(E-E_{i}\right) /\left(E_{b}-E_{a}\right)$ with $i=a, b$, respectively. In the above equations the variables with subscription $S$ indicate the values at the $R_{S}$. Thus, in the linear model the TA can be described by four parameters: $E_{a}, E_{b}, W_{0 s}$, and $V_{0 s}$. In general, these parameters except $V_{0 S}$ are determined by fitting the imaginary potentials with eq 1 , and the $V_{0 S}$ is obtained at the reference point of which the real potential is almost energy independent. Due to the energy limit, the present work lacks of some high energy points as the reference to extract the energy-independent potential $W_{0 S}$. Moreover, no elastic scattering data in the literature are available for this system. Anyway the real and imaginary potentials can be simultaneously fit by eq 1 and eq 2 , assuming the above equations are tenable (assuredly, this assumption is trustworthy). Thus the four parameters $E_{a}=89.2 \mathrm{MeV}$, $E_{b}=101.7 \mathrm{MeV}, W_{0 S}=-1.20 \mathrm{MeV}$, and $V_{0 S}=-2.19 \mathrm{MeV}$ were reliably extracted by the fits. The results are shown in Figure 3 with the solid lines.

3.3. Extract TA Parameters From Fusion Data. In fact, the energy dependent potentials can be extracted from the fusion excitation function. ${ }^{11}$ It is interesting to compare them with those from elastic scatterings. The BPM model with the consideration of nuclear deformation is used under the parabolic approximation. The partial cross section at each angle is given

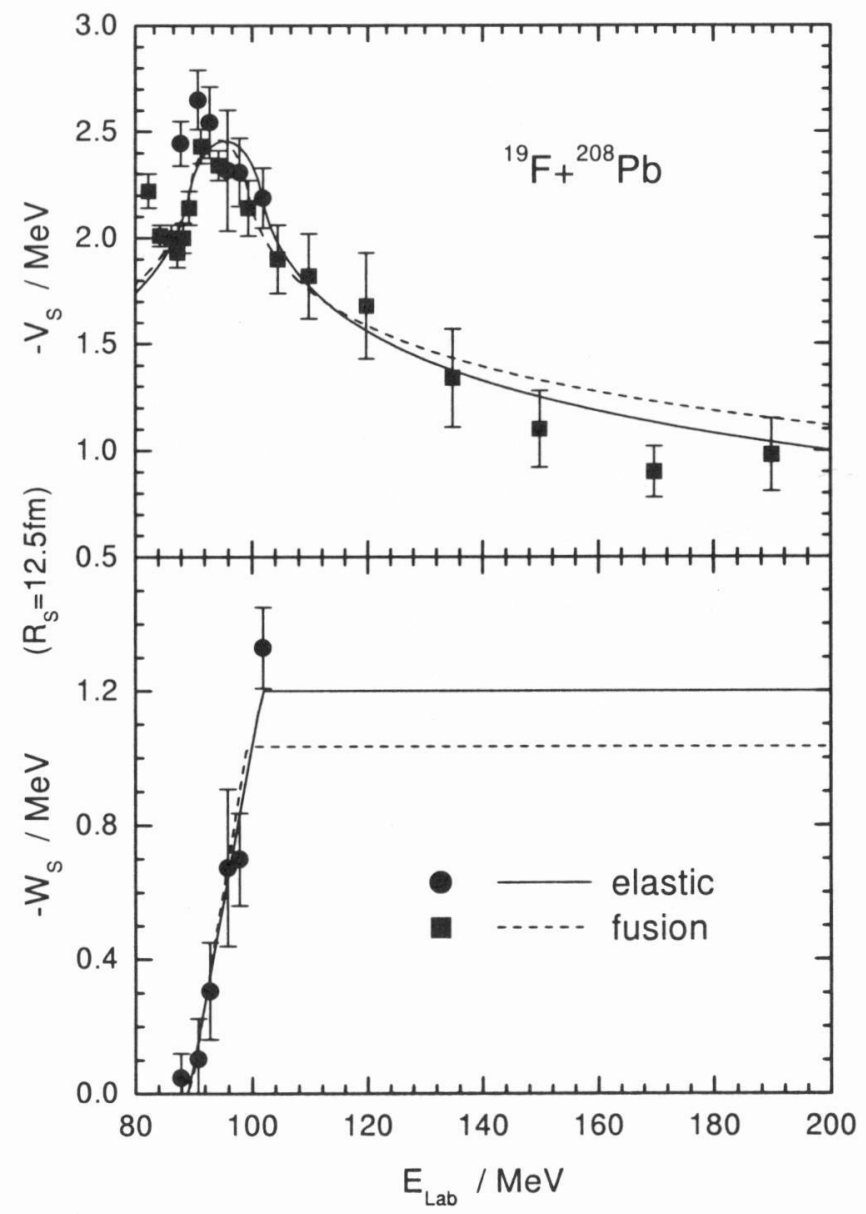

Figure 3. The real and imaginary potentials extracted from elastic and fusion channels. 
by

$$
\begin{array}{r}
\sigma_{f}^{l}(E, \theta)=\frac{\pi(2 l+1)}{k^{2}}\left[1+\exp \left\{\frac { 2 \pi } { \hbar \omega ( \theta ) } \left(V_{B}(\theta)-E_{\mathrm{cm}}\right.\right.\right. \\
\left.\left.\left.+\frac{l(l+1) \hbar^{2}}{2 \mu R_{B}^{2}(\theta)}\right)\right\}\right]^{-1},
\end{array}
$$

where $k$ is the wave number, $\mu$ is the reduced mass, $V_{B}(\theta), R_{B}(\theta)$, and $\hbar \omega(\theta)$ the barrier parameters (height, radius, and curvature) for different orientation, respectively. Summing over the partial waves, the above equation becomes

$$
\begin{aligned}
\sigma_{f}(E, \theta) & =\sum_{l} \sigma_{f}^{l}(E, \theta) \\
& =\frac{R_{B}^{2}(\theta) \hbar \omega(\theta)}{2 E_{\mathrm{cm}}} \ln \left[1+\exp \left\{\frac{2 \pi}{\hbar \omega(\theta)}\left(E_{\mathrm{cm}}-V_{B}(\theta)\right)\right\}\right] .
\end{aligned}
$$

Then the total cross section is given by integration over the angles

$$
\sigma_{f}(E)=\int_{0}^{\pi / 2} \sigma_{f}(E, \theta) d \theta
$$

On the basis of Wong model, ${ }^{12}$ the symmetrical deformations of ${ }^{19} \mathrm{~F}$ up to hexadecapole are taken into account in the calculations. The radial radius is expressed as

$$
R(\theta)=R_{0}\left[1+\beta_{2} Y_{2}(\theta)+\beta_{4} Y_{4}(\theta)\right] .
$$

Here $R_{0}$ is the radius of the spherical nucleus with equivalent volume, $\theta$ is the orientation angle of deformed nucleus with respect to the collision axis, and the $Y(\theta)$ is the spherical harmonic. The interaction potentials, including the Coulomb potential and the nuclear potential are taken as the deformed form,

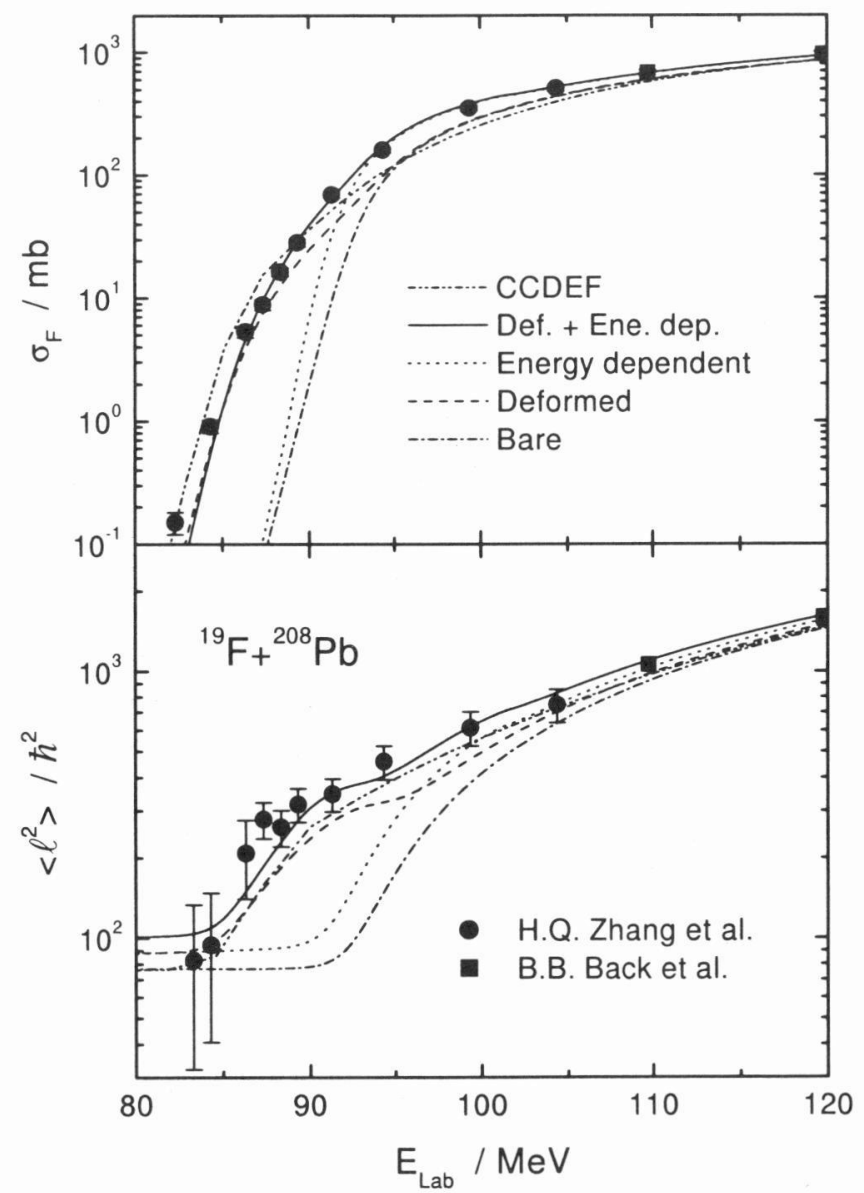

Figure 4. The fusion excitation functions and mean square spin distributions.

$$
\begin{aligned}
V_{C}(r, \theta)=\frac{Z_{1} Z_{2} e^{2}}{r}[1 & +\frac{1}{r^{2}} \sum_{i=P . T} R_{0 i}^{2}\left(\sqrt{\frac{9}{20 \pi}} \beta_{2 i} P_{2}\left(\cos \theta_{i}\right)\right. \\
& \left.+\frac{3}{7 \pi}\left[\beta_{2 i} P_{2}\left(\cos \theta_{i}\right)\right]^{2}\right) \\
& \left.+\frac{1}{r^{4}} \sum_{i=P . T} R_{0 i}^{4} \sqrt{\frac{1}{4 \pi}} \beta_{4 i} P_{4}\left(\cos \theta_{i}\right)\right] \\
V_{N}(r, \theta)=V_{0}[1+ & \exp \left\{\left(r-R_{0}\right.\right. \\
& -\sum_{i=P, T} R_{0 i}\left[\sqrt{\frac{5}{4 \pi}} \beta_{2 i} P_{2}\left(\cos \theta_{i}\right)\right. \\
& \left.\left.\left.\left.+\sqrt{\frac{9}{4 \pi}} \beta_{4 i} P_{4}\left(\cos \theta_{i}\right)\right]\right) / a\right\}\right]^{-1}
\end{aligned}
$$

with $R_{0}=R_{0 P}+R_{0 T}+0.29 \mathrm{fm}, R_{0 i}=\left(1.233 A_{i}^{1 / 3}-0.98 A_{i}^{-1 / 3}\right) \mathrm{fm}$, $i=P, T$, and $a=0.63 \mathrm{fm} . P(\cos \theta)$ is the Legendre polynomial whose orders are considered up to 4 , neglecting the higher order terms and other square terms. The nuclear potential only contains the real part for the fusion channel.

In terms of eq 3-8, $V_{0}$ can be determined by tuning it when the calculation value equals to the experimental fusion cross section. ${ }^{8,9}$ In order to compare with the TA behaviour of elastic scattering, the $V_{0 S}$ values are calculated at the same sensitive radius $12.5 \mathrm{fm}$. The results are also shown in Figure 3 with the square symbols. In the figure, the dashed line in the real part of potentials represents the best fit by eq 2 with TA parameters $E_{a}=89.5 \mathrm{MeV}, E_{b}=99.1 \mathrm{MeV}, W_{0 S}=-1.03 \mathrm{MeV}$, and $V_{0 S}=-2.23 \mathrm{MeV}$. The dashed line in the imaginary part of potentials is the results calculated by eq 1 . It should be pointed out

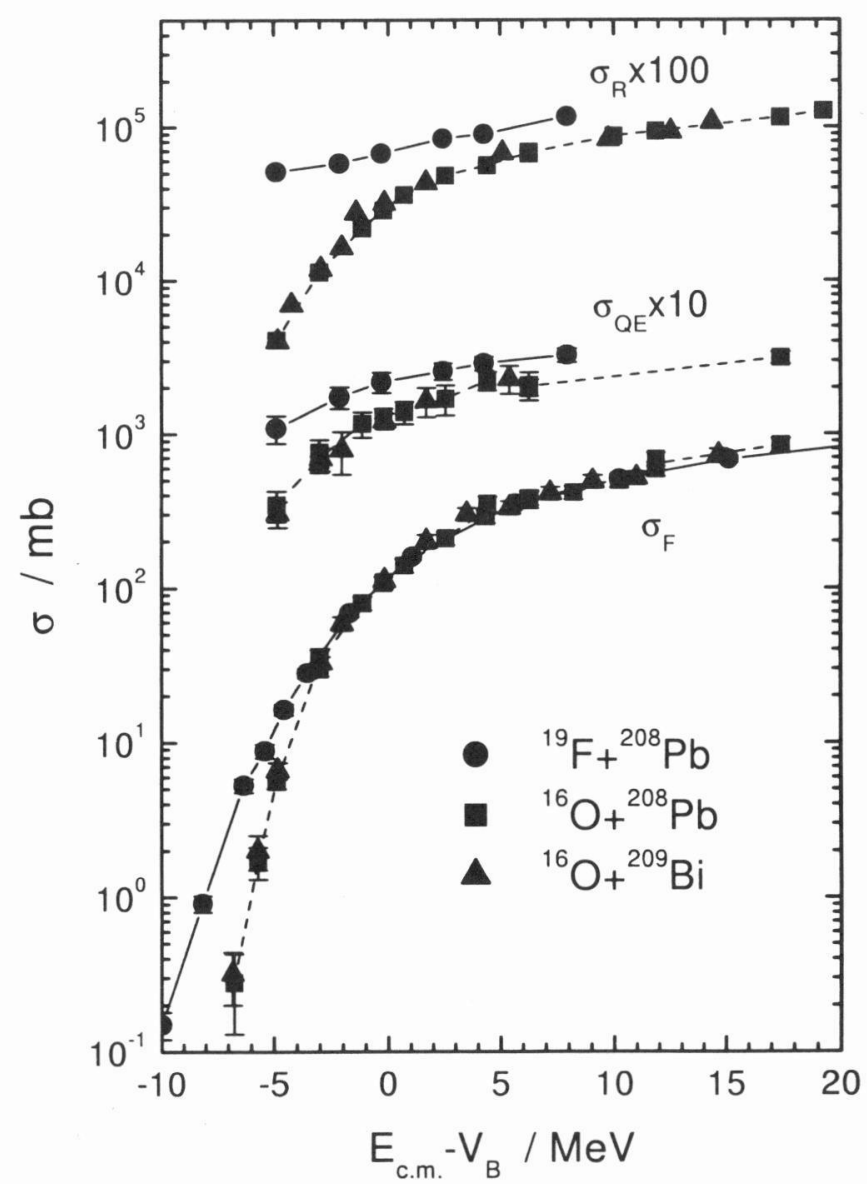

Figure 5. Comparison with the neighbouring systems. 
that the potentials extracted from the fusion data correspond to the inner radius and it has a large degree of uncertainty to extrapolate to an outer radius, for example, to the sensitive radius. Anyhow, it can be seen from the figure that these two kinds of TA behaviours are similar. This reflects that they have the same cause, namely $\mathrm{CC}$ effects.

\section{Discussion}

4.1. Fusion Excitation Function and Spin Distribution. In general, the energy dependent BPM model can well reproduce the enhancement of fusion cross sections near and below the barrier, but fails to describe the broadening of spin distribution. In addition to the energy dependence, the deformation effects are considered in the present work. The TA parameters extracted from elastic scatterings are employed in the calculations. The results well reproduce both cross sections and mean-square spins, as shown in Figure 4. The CC results calculated by CCDEF code are also illustrated in the figure for comparison. In the $\mathrm{CC}$ calculation, six inelastic channels are included, as done by Zhang et al., ${ }^{9}$ the $0.19714,1.3457,1.5540$, and $2.7798 \mathrm{MeV}$ excited states of ${ }^{19} \mathrm{~F}$ with $\beta_{2}=0.55, \beta_{3}=0.33$, $\beta_{2}=0.58, \beta_{4}=0.22$, as well as the 2.6146 and $3.1977 \mathrm{MeV}$ excited states of ${ }^{208} \mathrm{~Pb}$ with $\beta_{3}=0.12$ and $\beta_{5}=0.05$, respectively. It is clear that the increases of fusion cross sections and meansquare spins around the Coulomb barrier attribute to the energy dependent potential, in other words, to the dynamic polarization effects. While in the energy region further below the barrier, the static deformations of the nucleus should be considered to play an important role. The small bump around the barrier in the mean-square spin distribution results from the deformations.

4.2. Comparison with ${ }^{16} \mathrm{O}+{ }^{208} \mathrm{~Pb}$ and ${ }^{16} \mathrm{O}+{ }^{209} \mathrm{Bi}$ Systems. The reaction, quasi-elastic and fusion cross sections as a function of $E_{\mathrm{cm}}-V_{B}$ for these three systems are plotted in Figure 5. The data of ${ }^{16} \mathrm{O}+{ }^{208} \mathrm{~Pb}$ and ${ }^{16} \mathrm{O}+{ }^{209} \mathrm{Bi}$ systems are taken from References 5-7. Here the Bass barriers ${ }^{13}$ of $85.4 \mathrm{MeV}$, $77.1 \mathrm{MeV}$, and $78.0 \mathrm{MeV}$ are used for ${ }^{19} \mathrm{~F}+{ }^{208} \mathrm{~Pb},{ }^{16} \mathrm{O}+{ }^{208} \mathrm{~Pb}$, and ${ }^{16} \mathrm{O}+{ }^{209} \mathrm{Bi}$ systems, respectively. As shown in Figure 5, both reaction and quasi-elastic cross sections are comparable for the ${ }^{16} \mathrm{O}+{ }^{208} \mathrm{~Pb}$ and ${ }^{16} \mathrm{O}+{ }^{209} \mathrm{Bi}$ systems, while obviously larger for the ${ }^{19} \mathrm{~F}+{ }^{208} \mathrm{~Pb}$ system. Maybe, the larger probability of the quasi-elastic reactions results from the structure of projectile ${ }^{19} \mathrm{~F}$. The fusion cross sections are comparable for these three systems at near and well above barrier energies, but at energies lower than a certain value (i.e. $3 \mathrm{MeV}$ below the barrier) the fusion cross sections of ${ }^{19} \mathrm{~F}+{ }^{208} \mathrm{~Pb}$ system are obviously larger than those of the other two systems which are still comparable. It is difficult to understand why ${ }^{16} \mathrm{O}+{ }^{208} \mathrm{~Pb}$ and ${ }^{16} \mathrm{O}+{ }^{209} \mathrm{Bi}$ systems have the same tendencies in fusion excitation function while ${ }^{19} \mathrm{~F}+{ }^{208} \mathrm{~Pb}$ system does not. As shown in References 2,7 , the fusion excitation functions can be reproduced by consideration of the energy dependent potentials for ${ }^{16} \mathrm{O}+{ }^{208} \mathrm{~Pb}$ and ${ }^{16} \mathrm{O}+{ }^{209} \mathrm{Bi}$ systems. But for ${ }^{19} \mathrm{~F}+{ }^{208} \mathrm{~Pb}$ system, the above analysis shows that it is not enough to explain the larger fusion cross sections at low energies. When the effects of ${ }^{19} \mathrm{~F}$ deformations are also taken into account in the calculations, the fusion excitation function can be reproduced quite well. Hence, we may conclude that the nuclear deformations give rise to some effects in the process of fusion below the Coulomb barrier.

\section{Conclusion}

The angular distributions of elastic scatterings are presented at six energies near and below the Coulomb barrier for ${ }^{19} \mathrm{~F}+{ }^{208} \mathrm{~Pb}$ system. The phenomenological optical model analysis gives the depths of the real and imaginary potentials, showing that both real and imaginary potentials have pronounced energy dependence. The real parts of potentials derived from the fusion channel also show the energy dependence and are quite similar to those from the elastic channel. In the framework of the linear model, the TA parameters are extracted from both elastic and fusion channels. Comparison with the neighbouring systems shows that the projectile deformations have effects on the fusion reaction at low energy range. Using the TA parameters extracted from the elastic channels, the fusion excitation function and the mean-square spin distribution are reproduced by the deformed and energy dependent BPM model. In addition, the calculations indicate that the dynamic polarization effects play roles around the barrier, while the static deformations are at work at energies further below the barrier. From the above conclusion, we draw a deduction that the deformed configuration is of great benefit to synthesis of heavy or super heavy nuclide at low energy.

Acknowledgement. This work was supported by the $\mathrm{Nu}$ clear Industry Science Foundation of China under Contract No. 92A01010 and partly by the Major State Basic Research Development Program under Grand No. G200077400.

\section{References}

(1) C. Mahaux, H. Ngô, and G. R. Satchler, Nucl. Phys. A 449, 354 (1986).

(2) G. R. Satchler, Phys. Rep. 199, 147 (1991).

(3) H. Leucker, K. Becker, K. Blatt, W. Korsch, W. Luck, H. G. Völk, D. Fick, R. Butsch, H. J. Jänsch, H. Reich, and Z. Moroz, Phys. Lett. B 233, 277 (1989).

(4) D. R. Tilley, H. R. Weller, C. M. Cheves, and R. M. Chasteler, Nucl. Phys. A 595, 1 (1995).

(5) F. Videbæk, R. B. Goldstein, L. Grodzins, S. G. Steadman, T. A. Belote, and J. D. Garrett, Phys. Rev. C 15, 954 (1977).

(6) E. Vulgaris, L. Grodzins, S. G. Steadman, and R. Ledoux, Phys. Rev. C 33, 2017 (1986).

(7) P. Singh, S. Kailas, A. Chatterjee, S. S. Kerekatte, A. Navin, A. Nijasure, and B. John, Nucl. Phys. A 555, 606 (1993).

(8) B. B. Back, R. R. Betts, J. E. Gindler, B. D. Wilkins, S. Saini, M. B. Tsang, C. K. Gelbke, W. G. Lynch, M. A. McMahan, and P. A. Baisden, Phys. Rev. C 32, 195 (1985).

(9) H. Q. Zhang, Z. H. Liu, J. C. Xu, K. Xu, J. Lu, and M. Ruan, Nucl. Phys. A 512, 531 (1990).

(10) J. Raynal, Computing as a Language of Physics (IAEA, Vienna, 1972), p. 281.

(11) C. J. Lin, J. C. Xu, H. Q. Zhang, Z. H. Liu, F. Yang, and L. X. Lu, Phys. Rev. C 63, 064606 (2001).

(12) C. Y. Wong, Phys. Rev. Lett. 31, 766 (1973).

(13) R. Bass, Phys. Rev. Lett. 39, 265 (1977). 